\title{
A Survey on Multipacket Reception for Wireless Random Access Networks
}

\author{
Jia-Liang Lu, ${ }^{1}$ Wei Shu, ${ }^{1}$ and Min-You $\mathrm{Wu}^{2}$ \\ ${ }^{1}$ Department of Computer Science \& Engineering, Shanghai Jiao Tong University, Shanghai 200240, China \\ ${ }^{2}$ Department of Electrical \& Computer Engineering, The University of New Mexico, Albuquerque, NM 87131-0001, USA
}

Correspondence should be addressed to Jia-Liang Lu, jialiang.lu@sjtu.edu.cn

Received 24 March 2012; Revised 9 July 2012; Accepted 23 July 2012

Academic Editor: Yang Yang

Copyright (C 2012 Jia-Liang Lu et al. This is an open access article distributed under the Creative Commons Attribution License, which permits unrestricted use, distribution, and reproduction in any medium, provided the original work is properly cited.

\begin{abstract}
Multipacket reception (MPR) is the capability of simultaneous decoding of more than one packet from multiple concurrent transmissions. Continuous investigations on increasing the reception capability are giving new scientific contributions. In this paper, we provide an overview of MPR-related research work covering (1) the theoretically proved impacts and advantages of using MPR from a channel perspective to network capacity and throughput; (2) the various technologies that enable MPR from transmitter, transreceiver, and receiver perspectives; (3) previous work on protocol improvement to better exploit MPR. Indeed, MPR approaches have been applied in modern wireless mobile systems but the focus of this paper is to discuss MPR in random access wireless networks. Using MPR in such multihop environments calls for new adaptation on protocols, especially a cross-layer approach. To this end, we detail a scheduling method that targets full utilization of MPR capability.
\end{abstract}

\section{Introduction}

In the past decade, wireless technologies have become key technologies, offering mobile and flexible communications for industries, enterprises and individuals. Unlike a wired network where various kinds of physical connection, over copper, or fiber optics are used, nodes in a wireless network send information to each other sharing the common medium, air. An analogy to the problem is that people want to communicate with each other during a meeting in a room. The key is how to organize the conversations in this small room to let people get as much information as they can. This organization is known as medium access control. The most common assumption is that if several people talk at the same time, their voices will all be perturbed and communication will fail. There are two models for this common phenomenon. (1) From a channel-based point of view, the room is a single communication channel and the key is to enable multiple signals to occupy this channel. There are three basic types of techniques: Time-Division Multiple Access (TDMA), Frequency-Division Multiple Access (FDMA), and Code-Division Multiple Access (CDMA). (2) From a packet-based point of view, the room is the medium, and any simultaneous transmissions will cause collisions. Traditional medium access controls based on the collision model are viewed as collision recovery (e.g., Aloha), collision avoidance (e.g., CSMA/CA), or collision-free (Token Ring) techniques.

Different methods of analysis are used for different networks; a channel-based approach is often used for telecommunication systems such as wireless mobile systems, while a packet-based approach has been intensively investigated in computer networks such as wireless random access networks. However, the design of modern communicating systems involves both approaches. The signal processing techniques [3-7] in channel access enable a receiver to decode simultaneous signals from different transmitters. The notion of Multipacket reception (MPR) was also introduced in some of these works $[4,5]$ and is applied in telecommunication systems. It was in [6] that MPR was first discussed for the slotted Aloha protocol and for a random access wireless network.

Why does this have a large impact? MPR subverts the fundamental assumption of a wireless random access network, the collision model. Under the collision model, the capacity of a wireless network is limited mainly by the concurrent 
packet transmissions. Simultaneous transmissions lead to useless collisions and a significant degradation of network throughput, and retransmissions often make the situation even worse. All these disadvantages are related to the receiver capability of Single Packet Reception (SPR). But MPR can totally overturn the collision model by receiving the lost packet in a collision.

After several proposals on channel and medium access protocols on MPR $[6-8,22]$ were presented in the $90^{\prime} \mathrm{s}$, [23] was a seminal paper, the first to examine MPR, as an interaction between the physical and medium access control layers for a wireless random access network. The MPR node model used in [23] was derived from [6]. The MPR capability of a node is modeled by an MPR matrix with conditional probability $R_{n, k}$ that $k$ packets are correctly received given that $n$ packets are transmitted.

$$
R=\left(\begin{array}{ccccc}
R_{1,0} & R_{1,1} & & & \\
R_{2,0} & R_{2,1} & R_{2,2} & & \\
R_{3,0} & R_{3,1} & R_{3,2} & R_{3,3} & \\
\vdots & \vdots & \vdots & \vdots & \ddots
\end{array}\right) .
$$

The fundamental change of this model compared to the collision model is that the reception can be described by conditional probabilities instead of deterministic failure when simultaneous transmissions occur.

An interesting observation on the recent work on MPR is that significant results have been obtained on the capacity and throughput analysis based on the MPR model, while the enabling MPR technologies are rarely mentioned in these papers. Many studies are based on an assumption that receivers are able to extract, in some optimal way, the signals from multiple transmitters despite the interference with one another. We find an increase of confusion in MPR techniques and how to use MPR techniques. We think that it comes from the gap of channel-based approach and packet-based approach.

One of the purposes of this survey is to clarify MPR enabling techniques by classifying them into transmitter, trans-receiver, and receiver perspectives (Section 3 ). We expect this survey would help researchers working in related areas to make more realistic hypothesis with the MPR model. The second purpose is to discuss how to use MPR. MPR can bring significant improvement in capacity and throughput (Section 2) but it needs to cope with MAC and the networking layer to achieve real improvement. We will discuss major work on MAC and networking protocol design to exploit MPR (Section 4) and in the same part we detail a scheduling method that targets full utilization of MPR capability using a cross-layer approach.

\section{Impacts of MPR}

The MPR matrix given in (1) describes the capability of a receiver through a channel. The weakest MPR is equivalent to a conventional collision channel $R_{0}$, while the strongest
MPR $R_{1}$ corresponds to the case that all transmitted packets are perfectly received and separated:

$$
\begin{aligned}
& R_{0}=\left(\begin{array}{llllll}
0 & 1 & & & \\
1 & 0 & 0 & & \\
1 & 0 & 0 & 0 & \\
\vdots & \vdots & \vdots & \vdots & \ddots
\end{array}\right), \\
& R_{1}=\left(\begin{array}{llllll}
0 & 1 & & & \\
0 & 0 & 1 & 0 & \\
0 & 0 & 0 & 1 & \\
\vdots & \vdots & \vdots & \vdots & \ddots
\end{array}\right) .
\end{aligned}
$$

In between, the form of the MPR matrix depends on the channel conditions, capture models, and signal separation algorithms [23]. This MPR matrix was initially proposed to model a multiple reception channel and today it is the usual model for a receiver from the packet access point of view.

2.1. One-Hop Throughput. Let's first discuss the throughput on a node. In [8], the number of successfully received packets in a time slot is defined as the one-hop throughput. It is the MPR channel capacity from the channel-based point of view. It is easy to understand that when we take an arbitrary node, the number of packets that are allowed to go through the node describes exactly the receiver channel characteristics. The average throughput is upper bounded by

$$
\eta=\sup _{i=1}^{n} R_{n}
$$

where

$$
R_{n}=\sum_{k=1}^{n} k R_{n, k}
$$

$R_{n}$ is the expected number of correctly received packets given that $n$ packets are transmitted. $\eta$ is defined as the capacity of the MPR channel.

The above statement is validated under saturation traffic. When we consider a Poisson distribution of the intervals between packet arrivals, the following formula should be considered:

$$
\eta_{\text {Aloha }}=\sup _{\lambda \geq 0} e^{-\lambda} \sum_{i=1}^{n} \frac{\lambda^{i}}{i !} R_{i} .
$$

The maximum stable one-hop throughput of a MPR node with $n$ neighbors using Aloha-like protocols is given by [6]. According to (5), the maximum one-hop throughput of the conventional collision model can be obtained as $\eta_{\text {Aloha }}=$ $\sup _{\lambda \geq 0} e^{-\lambda} \lambda$ by taking $R_{1}=1$ and $R_{i}=0, i>1 . R_{i}$ is therefore the only factor that impacts the one-hop throughput in MPR model over conventional collision model.

2.2. Spatial Throughput. The spatial throughput is used to move the focus from one arbitrary node to a packet radio network node. In [9], the authors consider such a network with nodes spatially distributed in the plane according to a 
Poisson process with parameter $\lambda$. A set of assumptions is adopted from [4] to extend the spatial throughput analysis to networks with MPR nodes.

Given the probability $p$ of a node transmitting at a given time slot (in Slotted Aloha), the probability that one node $r$ receives $x$ packets is

$$
P[X=x]=P[X=x \mid A] P[A],
$$

where $A$ is the event that $r$ does not transmit; therefore $P[A]$ equals $(1-p) . P[X=x \mid A]$ depends on the probability $P[T]$ of the event $T$ that a sender $t$ transmits in the time slot and the density of the senders, hence the following expression:

$$
\begin{aligned}
P[X=x \mid A]= & \sum_{n=0}^{\infty} \sum_{k=x}^{n}\left(\begin{array}{l}
k \\
x
\end{array}\right) P[T]^{x}(1-P[T])^{k-x} \\
& \times R_{n, k} \frac{\left(\lambda p \pi r_{0}^{2}\right)^{n}}{n !} e^{-\lambda p \pi r_{0}^{2}},
\end{aligned}
$$

where $r_{0}$ is the transmission range and $R_{n, k}$ is the conditional probability in the MPR matrix (1). According to Equations (6) and (7), the probability distribution function of $X$ can be obtained. The spatial throughput on receiver $r$ is immediately obtained by taking the expectation of $X$ :

$$
E[X]=\sum_{x=1}^{\infty} x P[X=x] .
$$

If there are $N$ nodes in the network, the local throughput, $S$, of the network is

$$
S=N E[X]
$$

The spatial local throughput with different values of $k$ is summarized in Figure 1, in which we observe as expected that the MPR feature improves the maximum achievable local throughput. The improvement ratio is close to a liner function of $k$.

2.3. Capacity. Since 2007, a series of results on capacity improvement by protocol architectures that exploit MPR has been published in [10-12]. Extensive results for $k$-MPR and a combination of MPR, multichannel, and multiinterface can be found in $[13,24]$. Hereafter, $\Theta, O$, and $\Omega$ stand, respectively, for a tight bound, upper bound and lower bound.

Given $n$ as the number of nodes in the network, GarciaLuna-Aceves et al. [10] have shown that a 3D random MPRbased wireless network has a capacity gain of $\Theta(\log n)$. And MPR is proved to achieve a better capacity improvement for wireless ad hoc networks than Network Coding (NC) when the network experiences a single-source multicast and multipair unicasts.

Also assuming that all the transmissions within receiving range can be decoded, they [11] further prove that MPR does increase the order of the transport capacity of random wireless ad hoc networks for multi-pair unicast applications by a factor of $\Theta(\log (\log n))$ under the SINR (Signal to Interference plus Noise Ratio) model.

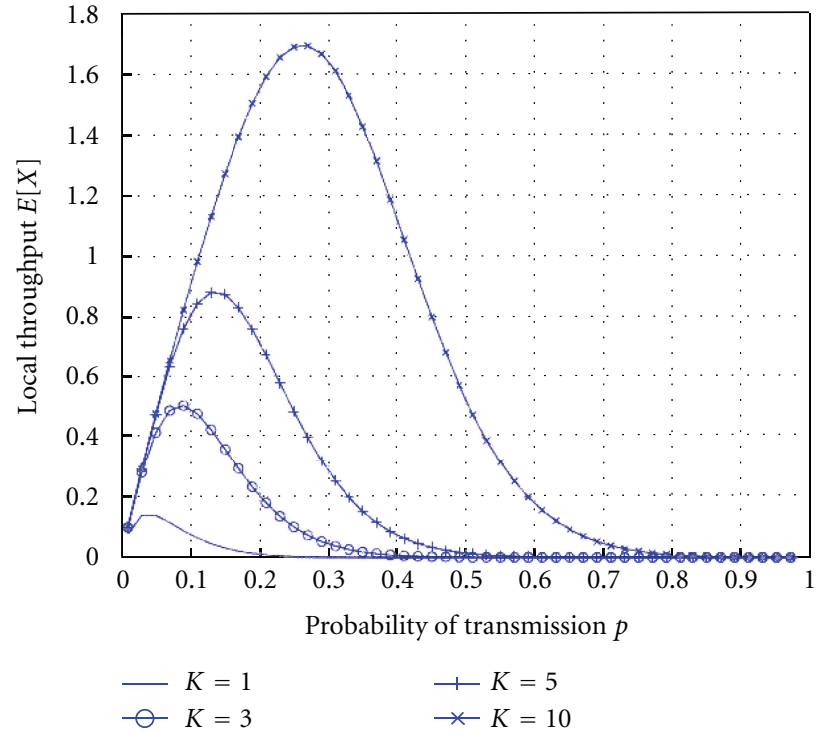

FIGURE 1: Local throughput in packets/timeslot for the simple model of multipacket.

In [12], energy efficiency is taken into account as a factor to increase the capacity of the network. They showed that $\lambda(n)=\Theta\left(R(n)^{(1-2 / \alpha)} / n^{1 / \alpha}\right)$ bits per second constitutes a tight upper and lower bound for the throughput capacity of random wireless ad hoc networks, where $\alpha>2$ is the path loss parameter in the physical model, $n$ is the total number of nodes in the network, and $R(n)$ is the MPR receiver range. The MPR achieves a capacity gain of at least $\Theta\left((\log n)^{((\alpha-2) / 2 \alpha)}\right)$ when $R(n)=\Theta(\sqrt{\log n / n})$ compared to Gupta and Kumar's result.

Several important results on the improvement of $k$-MPR over collision-based wireless networks (1-MPR) are given in [13].

(i) For an arbitrary $k$-MPR network, the capacity gain over 1-MPR networks is $\Theta(\sqrt{k})$, when $k=O(n)$.

(ii) The capacity is $\Theta\left(W_{n}\right)$ bit-meter/sec and the network is scalable, when $k=\Omega(n)$.

(iii) For random $k$-MPR networks, the capacity upper bound and lower bound match and the capacity gain over 1-MPR networks is $\Theta(k)$ when $k=O(\sqrt{\log n})$.

(iv) Even the lower bound has a capacity gain of $\Theta(\sqrt{\log n})$ when $k=\Omega(n)$.

In [25], Wang et al. summarize their work on MPR by presenting a unifying approach for the computation of throughput capacity of wireless ad hoc networks under all data traffic patterns including unicast, multicast, broadcast, and anycast. They use an $(n, m, k)$-cast formulation, where $n, m$, and $k$ denote the number of nodes in the network, the number of destinations for each communication group, and the actual number of communication group members that receive the information, respectively. The protocol model in [11] is used. They show that the per source-destination 
( $n, m, k)$-cast throughput capacity $C_{m, k}(n)$ is tightly bounded by $\Theta(R(n) \sqrt{m} / k), \Theta(1 / k)$, and $\Theta\left(R^{2}(n)\right)$ w.h.p when $m=$ $O\left(R^{-2}(n)\right), \Omega(k)=R^{-2}(n)=O(m)$, and $k=\Omega\left(R^{-2}(n)\right)$, respectively. The most important fact that they proved is that for the minimum value of the received range $(R(n)=$ $\Omega(\sqrt{\log n / n}))$ required to guarantee network connectivity; the $(n, m, k)$-cast throughput capacity with MPR has a gain of $\Theta(\log n)$ compared to the throughput attained with SPR. In Table 1, we recapitulate the theoretical capacity results related to $\mathrm{MPR}$.

2.4. Stability. The majority of capacity and throughput calculations assume that the network is saturated. It is very important in this condition to analyze the stability. The stability relies on whether a maximum stable throughput is reachable, that is, whether the probability of packet buffer overflow can be made arbitrarily small by making the buffer size sufficiently large. In [5], Ghez et al. give two important theorems for the Slotted Aloha network with MPR,

(1) If $R n$ has a limit $R=\lim _{n \rightarrow \infty} R_{n}$, then the system is stable for all arrival distributions such that $\lambda<R$ and is unstable for $\lambda>R$.

(2) The system is stable for $\lambda<\lim _{n \rightarrow \infty} R_{n}$ and unstable for $\lambda>\lim \sup _{n \rightarrow \infty} R n$.

In [26], a general asymmetric MPR model is introduced and the medium-access control (MAC) capacity region is specified. It is shown that the stability region undergoes a phase transition from a concave region to a convex polyhedral region as the MPR capability improves in a twouser system. Furthermore, it is shown that persistent Aloha is also optimal for the symmetric MPR model in a wide range of MPR regimes.

From these two theorems, it is clear that if the number of simultaneous transmissions is greater than the average MPR capacity, the system cannot achieve a maximum stable throughput. Therefore, to exploit MPR, scheduling should still be used among the transmitters. Some recent papers which begin to address this problem $[2,19]$ will be discussed in Section 4.2.

Dua [27] analyzed a one-dimensional Markov chain which captures the evolution of the queue of a typical transmitter in isolation in an MPR system. Both static channels under ideal power control and fading channels under ideal slow power control are studied. He provides sufficient conditions under which the system converges to a unique steady state. And the average delay per packet for an MPR network is given by

$$
D=\frac{\eta-\Delta \cdot p}{p(\Delta-\eta)},
$$

where $\eta$ is the effective probability of transmission for a transmitter in steady state $\Delta$ is the actual probability for a transmitter with a nonempty queue.

In $[28,29]$, the analytical equations for the characteristics of a relay node's queue such as average queue length and stability conditions are studied for the throughput per user.
The authors consider a network with $N$ users sources, one relay node with MPR, and a single destination node with MPR as well. By modeling the relay node's queue with a onedimension Markov Chain, they show that the throughput with $N$ source nodes with a relay node is $N$ times the throughput without a relay: $\mu_{\text {total }}=N \mu$, and the throughput is stable if the transmission probability of the relay is bigger than a reference probability $Q_{0 \text { min }}$ that can be computed with the stability condition of the Markov chain.

\section{How to Realize MPR}

Significant improvements to both throughput and capacity of a wireless network can be obtained with MPR. There are a number of techniques which allow simultaneous decoding of packets on a receiver but in many papers MPR is said to be realized with CDMA or MIMO. The appearance of these notions together with MPR brings some ambiguities to the understanding of MPR enabling techniques. Examples such as CDMA and MIMO cover a wide range of techniques in signal processing and wireless systems. Give the current research achievements around MPR, it is worthwhile to set up a clear classification of MPR technologies and to exclude misleading notions. To this end, we give a classification based on transmitter perspective, trans-receiver perspective, and receiver perspective.

3.1. Transmitter Perspective. The first class of techniques that enable MPR require a significant effort by the transmitter. Examples such as CDMA and OFDMA fall into this class. CDMA allows multiple users to be multiplexed over the same wireless channel by employing a coding scheme where each transmitter is assigned a code. The baseband signal is multiplexed with a spreading code running at a much higher rate. The spreading code is a pseudorandom code, and all codes used for one channel are orthogonal. Therefore, on the receiver side, an unwanted signal will be eliminated by the cross-correlation decode, and only the relevant signals are conserved. This technique allows the receiver to decode multiple data streams with the different codes that are known a priori. The ability to decode multiple data packets depends on the selection of code. For example, the orthogonality is the key that allows the receiver to decode the set of simultaneous arrived signals, and this is done on the transmitter side.

OFDMA competes with CDMA as a major multiaccess technique. It is used to increase the wireless channel efficiency based on multicarrier modulation methods (in IEEE802.11 a,g,n). For each packet, several data streams are transmitted over the subcarrier frequencies as many slowlymodulated narrow-band signals rather than one rapidlymodulated wide-band signal. In OFDMA, the transmitters of a receiver are assigned different OFDM subchannels. Since the sub-channels do not interfere with each other, MPR is enabled on a frequency basis. In [30], the authors describe a many-to-many communication in which the transmissions are divided in frequency to allow the receiver to decode the packets. Again, OFDMA enables MPR with a great effort for the transmitter in sub-channel selection. Furthermore, it 
TABLE 1: Theoretical results on the capacity.

\begin{tabular}{|c|c|c|c|c|}
\hline Metric & Condition & SPR & MPR & Works \\
\hline One-hop throughput (upper bound) & Slotted aloha & $\sup _{\lambda \geq 0} e^{-\lambda}$ & $\sup _{\lambda \geq 0} e^{-\lambda} \sum_{i=1}^{n} \frac{\lambda^{i}}{i !} R_{i}$ & {$[8]$} \\
\hline Spatial throughput & Slotted aloha & $\sum_{x=1}^{\infty} P[X=x]$ & $P[X=1]$ & {$[9]$} \\
\hline Throughput (tight bound) per source-destination & Protocol model (graph model) & $\Theta\left(\sqrt{\frac{1}{n}}\right)$ & $\Theta\left(\sqrt[3]{\frac{\log n}{n}}\right)$ & [10] \\
\hline Throughput (lower bound) per source-destination & Physical model (SINR model) & $\Theta\left(\frac{1}{\sqrt{n \log n}}\right)$ & $\Theta\left(\frac{\log (\log n)}{\sqrt{n \log n}}\right)$ & [11] \\
\hline Throughput (tight bound) per source-destination & Multiflow SINR model & $\Theta\left(\sqrt{\frac{1}{n}}\right)$ & $\Theta\left(\frac{(\log n)^{(1 / 2)-(1 / \alpha)}}{\sqrt{n}}\right)$ & {$[12]$} \\
\hline Network throughput (tight bound) & $k$-MPR (arbitrary networks) & $\Theta(\sqrt{n})$ & $\begin{array}{c}\Theta(\sqrt{k n}), k=O(n) \\
\Theta(n), k=\Omega(n)\end{array}$ & {$[13]$} \\
\hline Network throughput (lower bound) & $k$-MPR (random networks) & $\Theta\left(\sqrt{\frac{n}{\log n}}\right)$ & $\begin{array}{c}\Omega\left(k \sqrt{\frac{n}{\log n}}\right), k=O(\sqrt{\log n}) \\
\Omega(\sqrt{n}), k=\Omega(\sqrt{\log n})\end{array}$ & {$[13]$} \\
\hline
\end{tabular}

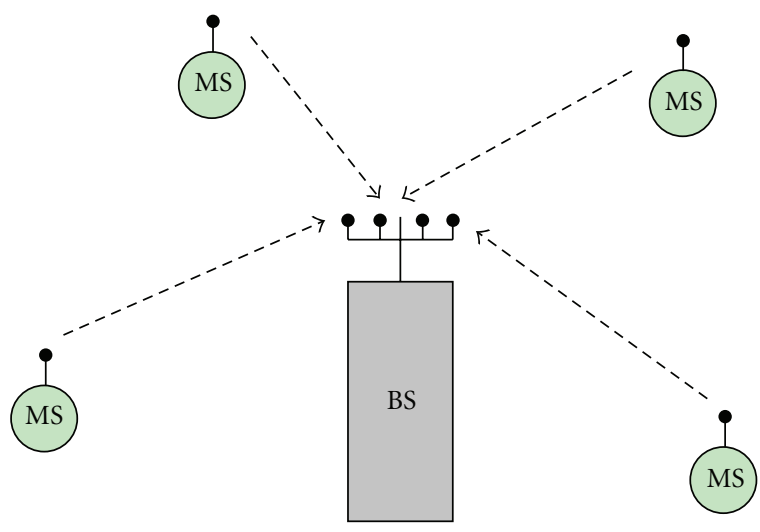

FIgUre 2: The BS is MPR in a MIMO system with multiple antennas.

is a pseudo-MPR capability, because the bandwidth is also divided at the same time, when the radio channel is divided into sub-channels.

3.2. Transreceiver Perspective. In this class of techniques, to enable MPR, transmitters and receivers should cooperate on some operations. Multiantenna MIMO system [14] can achieve MPR by exploiting the spatial diversity of the transmissions. In such a system, each antenna corresponds to a different channel characteristic $H(t)$. A packet sent through one antenna can be easily distinguished from one sent with another antenna by channel estimators. In case of a cellular or AP-based wireless network, the communication between Mobile Station (MS) and Base Station (BS) is based on the MPR capability of the BS (Figure 2). The realization of MPR in a multi-antenna MIMO system requires both transmitters and receivers to implement specific functionalities. That is how it differs from pure transmitter-based techniques.

Another type of technique that could in principle be used for MPR is signal separation. The problem of packet separation can be formulated as one of signal separation in a MIMO system. In [31], the authors present Known
Modulus Algorithms (KMA) to allow packet separation in asynchronous ad hoc networks. In the algorithm, a transmitter needs to send a constant modulus signal multiplied by an amplitude modulating code known at the receiver and an antenna array is used on the receiver side which can detect and filter out the desired user among the other interfering signals with the help of this modulation code. The modulation code can be a random binary sequence determined either by the transmitter or the receiver. In [32], a variation of KMA, Algebraic KMA (AKMA), is proposed based on a matrix perturbation analysis. Other signal separation techniques based on a Constant Modulus Algorithm (CMA) or Multiple Modulus Algorithm (MMA) [33] require blind equalization and are usually not efficient for MPR.

In [34], the authors propose to use polynomial phasemodulating sequences to provide MPR capability. The algorithm exploits the fact that the baseband signal exhibits cyclostationarity properties, which are induced at the transmitters after modulation with polynomial phase sequences. So the proposed modulation does not expand the bandwidth and can be considered as a color code to distinguish packets from different users.

Some resource allocation base techniques fall also in this class. It is mainly based on a network-assisted approach. The network-assisted diversity is firstly introduced in [35] as a technique to separate the collided packets. The collided packets are kept in memory rather than being discarded and are later combined with future retransmissions to extract all the collided information packets. The proposed method is suitable for multiplexing variable-bit-rate sources without affecting the physical layer bit rate parameter of each source. In [36], a bit-map-assisted dynamic queue (BMDQ) protocol is presented. In the proposed protocol, the traffic in the channel is viewed as a flow of transmission periods, each of which has a bit-map slot for user detection so that accurate knowledge of active users can be obtained. To summarize, resource allocation reuses signal processing principles on packet level. 
3.3. Receiver Perspective. Next, we present a class of techniques that involve only the receiver for decoding several packets simultaneously. Compared to the previous two classes, this class of solutions comes closer to the ideal of MPR, to shift the responsibility from transmitters to receivers.

The Match Filter (MF) approach is widely used for single user detection. Even though it is not optimal when multiple users are present, still a receiver can use a bank of Match Filters [9] to decode packets coded with spreading codes that need not even be orthogonal.

Techniques used to separate signals for Multiuser Detection (MUD) are more applicable for MPR. That is why many papers [2, 10-12] on network capacity with MPR cited it as the technique to realize MPR. It is a way to alleviate Multiple Access Interference (MAI) during the simultaneous transmissions on the same channel.

An optimal MUD detector refers to maximum likelihood sequence estimation (MLSE) [37] which requires knowledge of all transmitters' spreading codes (e.g., base stations in a cellular CDMA system). This optimal detector is too complicated for practical application although it has excellent performance. One of the reasons, given in [38], is that cellular system is centrally controlled and always has synchronization among different users to some extent. However, in distributed wireless networks, it is quite difficult to apply signal processing techniques to separate the asynchronous transmissions. Therefore, MLSE is not a candidate for MPR.

In suboptimal MUD techniques, two approaches can be identified, namely, linear and nonlinear MUD. In linear MUD, a linear transformation is applied to the soft outputs of the conventional detector in order to produce a new set of decision variables with MAI greatly decoupled. Two most cited linear multiuser detectors are decorrelated detectors [39] and the Minimum Mean Square Error (MMSE) detector [9]. They are generally complex but yield an optimal value of the near-far resistance performance metric.

On the other hand, non-linear MUDs use interference estimators and remove the interference from the received signal before detection. They are much simpler but have an inferior performance compared to linear MUD. Multistage Interference Cancellation (IC) is one of the most interesting in this category, where cancellation can be carried out either successively (SIC) or in parallel (PIC).

For SIC, the multi-user's signals are demodulated and cancelled from the strongest to the weakest according to their received signal power. References [2, 10-12] assume the receiver node utilizes MUD and SIC to decode multiple packets. It is a misleading phrase, because SIC is a method in MUD. Indeed, it may be the most practical way to realize MPR given the current state of the art.

For PIC, without the exact knowledge of the interfering bits, their estimates in the previous stage are used instead. To enhance the performance of PIC, a multistage approach is often adopted, in which the detector at $n$th stage uses the bit decision from the $(n-1)$ th stage. In theory, PIC could support more simultaneous packets from different users, but a perfect power control is necessary. In practice, it is shown in [40] that in a single of a 2-path Rayleigh fading channel, 2-stage PIC, and SIC have very close performance.

In Figure 3, the techniques that are applied for MPR are summarized with a tree classification. Again, many of these techniques were applied to mobile communication systems such as cellular networks; for wireless random access network only a subset of techniques is possible due to the distributed random channel access nature. Among the three classes, our view is that the receiver-based techniques are more applicable for wireless random access networks. They also meet the objective of the introduction of MPR in a wireless random access network, which is shifting the responsibility from the transmitters to the receivers.

3.4. Enabling MPR in a Wireless LAN. The techniques described in the previous section work for random access wireless networks. However, there is a class of technologies which are specific to WLANs based on an access point (AP).

In [1], Zheng et al. consider the uplink MPR in a WLAN, that is, from client station to AP. The AP has M antennas, while each client station has only one antenna Figure 4.

Based on this model, Zhang et al. investigate a number of topics on MPR in WLANs: [41] gives a delay analysis; [42] studies the impact of capacity improvement with MPR in a WLAN; [43] proposes a multiround contention randomaccess protocol to increase the channel utilization; [44] extends the investigation to the nonsaturation case and shows that super-linear scaling also holds for two newly defined situations, networks with with bounded mean delay, and bounded delay jitter. The focus of this survey is general wireless random access networks; therefore, the above works focusing particularly on WLANs will not be further detailed.

In [14], an MPR-based MAC protocol with adaptive resource allocation is proposed for a MIMO/OFDM-based WLAN. The idea of MPR is applied through a request-tosend/clear-to-send-(RTS/CTS-) based MAC protocol along with MUD to resolve the collision problem. A realistic collision model is employed, taking into consideration the PHY layer parameters such as channel information, spacetime-coded beam-forming, and multiuser detection, as well as subcarrier, bit, and power allocation. Several numerical results show that this PHY-MAC cross-layer optimization enhances the throughput of the system, because MPR can greatly reduce the probability of collisions.

In [15], contention resolution algorithms are proposed for MPR in a slotted Aloha WLAN. These algorithms require centralized information, the retransmission probability computed by the base station. Based on this information, the algorithm chooses the optimal retransmission probability to maximize the expectation of the system throughput conditioned on the number of retransmitting terminals.

Table 2 lists the realization of MPR in a WLAN.

\section{Protocols with MPR}

As discussed in the previous section, a set of technologies enable MPR capability for wireless nodes, and each has easy and hard parts in practice. MPR has also been proven to 


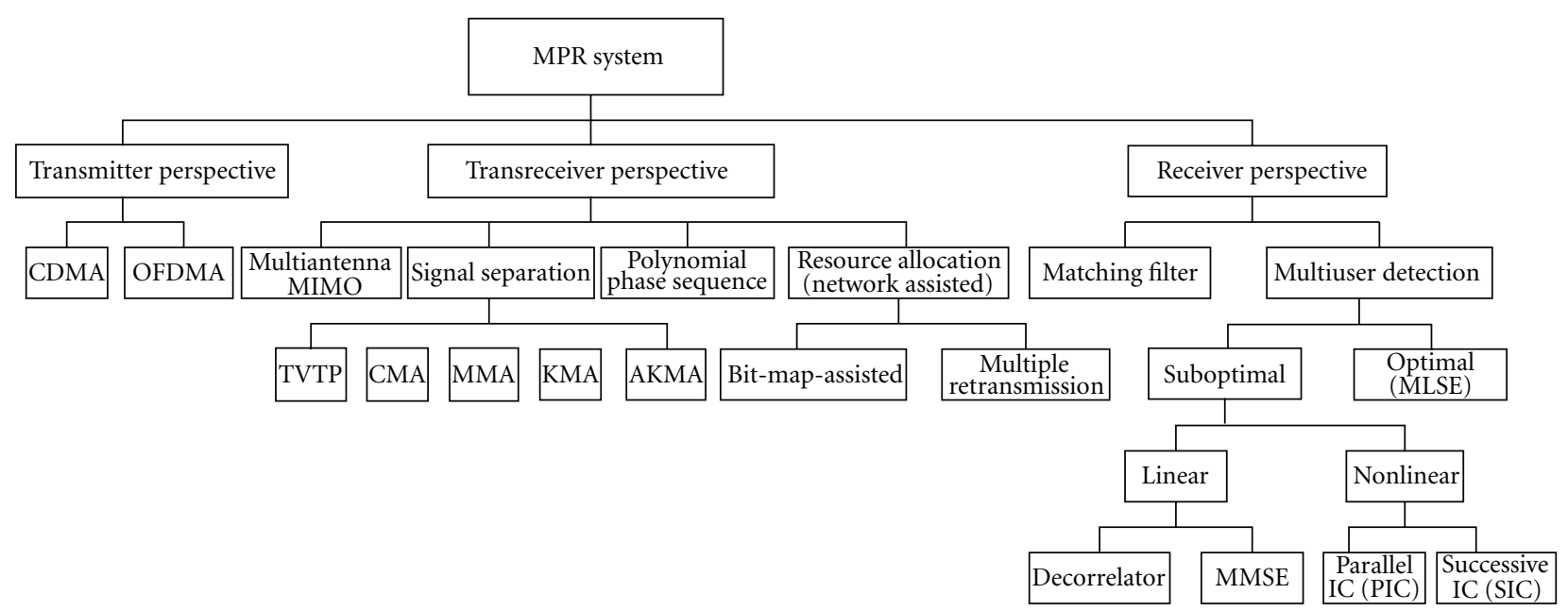

FIgURE 3: Classification of techniques applied for MPR.

TABLE 2: WLAN with MPR.

\begin{tabular}{lllll}
\hline Protocol & MPR realization & MPR adaptation & Performance results & Works \\
\hline Zheng's & Multiple antennas on AP & Modified CTS & Maximum throughput scale with $k(k$-MPR) & {$[1]$} \\
Huang's & MIMO with multiple antennas & Allocation scheme & 5.2 packets/ms (with MIMO) & 3.1 packets/ms (SPR) \\
DFT & \multirow{2}{*}{ Spreading-centralized control by AP } & Contention resolution & $77.3 \%$ of theoretical throughput \\
IFT & & & $69.7 \%$ \\
\hline
\end{tabular}

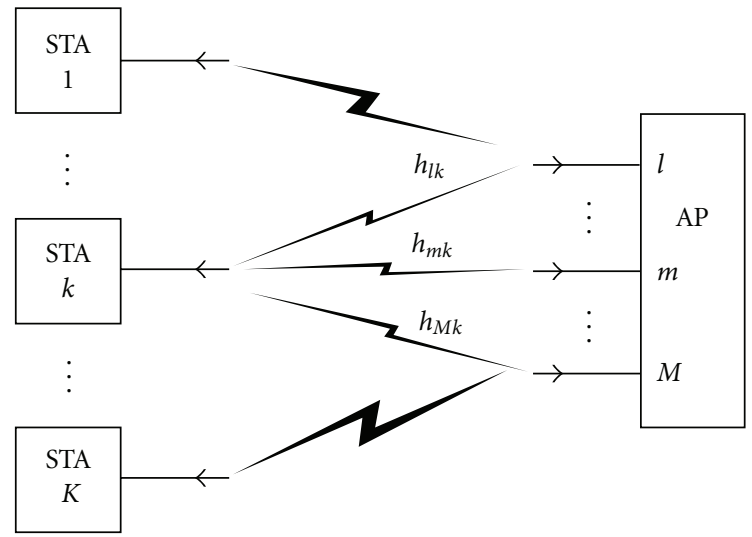

FIgURE 4: System configuration for uplink MPR in [1].

improve the network capacity in various works reviewed in Section 2. At the same time, MPR introduces greater challenges in network protocol design, and it requires closer interactions between the layers of the wireless network. We consider the design and analysis of MAC and networking protocols which employ adaptations to fully utilize MPR capability.

4.1. MAC with MPR. Mergen and Tong [8] proposed a multiple-access protocol based on receiver controlled transmission (RCT). RCT is a hybrid scheduled and random access MAC, which applies scheduling to determine the receiving nodes and then the transmitters for each receiving node. RCT aims to maximize local throughput by granting an appropriate subset of users so that the varying levels of MPR capability are exploited. The simulation results show that using RCT with MPR achieves almost double the local throughput of the slotted Aloha protocol. Although this first work pointed out that adaptation on the MAC layer is necessary to fully exploit MPR capability, the proposed scheduling works with predefined network topologies and is hard to extend to general ad hoc networks.

Later in [16], Zhao and Tong designed a multiqueue service room (MQSR) protocol, which exploits MPR capability to handle users with different quality of service constraints. For each slot, the number of users is computed to maximize the expected number of successfully received packets. The performance of MQSR is compared with slotted Aloha and URN [3] with 2-MPR. The URN protocol manages to optimally adapt to the network load. In Figure 5, both MQSR and URN can extend the network capacity. But MQSR approaches the maximum throughput upon the transmission probability $P=0.5$, while URN and slotted Aloha achieve their maximum throughput when $P$ close to 1 .

$\mathrm{Yu}$ and Giannakis associate a contention tree algorithm with SIC to propose SICTA in [45]. They aim to create a collision resolution access protocol, from the packet point of view. It turns out to be an interesting approach for MPR. Performance metrics including throughput and delay are analyzed to establish that SICTA outperforms existing contention tree algorithms reaching 0.693 in stable throughput.

Celik et al. point out in $[17,46]$ that the near-far problem reminds an important factor which degrades the throughput and the fairness in a wireless network even with 
TABle 3: MAC with MPR.

\begin{tabular}{llll}
\hline Protocol & MPR adaptation & Performance results & Works \\
\hline RCT & Scheduling, reception feedback & 4.7 packet/slot $(\mathrm{MPR})$ & 2.5 packet/slot $(\mathrm{SPR})$ \\
& & Max throughput with $P=0.5(\mathrm{MPR})$ & {$[8]$} \\
MQSR & Estimation of the state of users & Max throughput with $P=1$ (optimal SPR) & {$[16]$} \\
GDP & Switching between two transmission probabilities & Improved overall throughputand fairness & {$[17]$} \\
CMGPQ & Cooperative multigroup priority queuing & $30 \%$ improvement on throughputunder light load $(P<0.6) \quad[18]$ \\
\hline
\end{tabular}

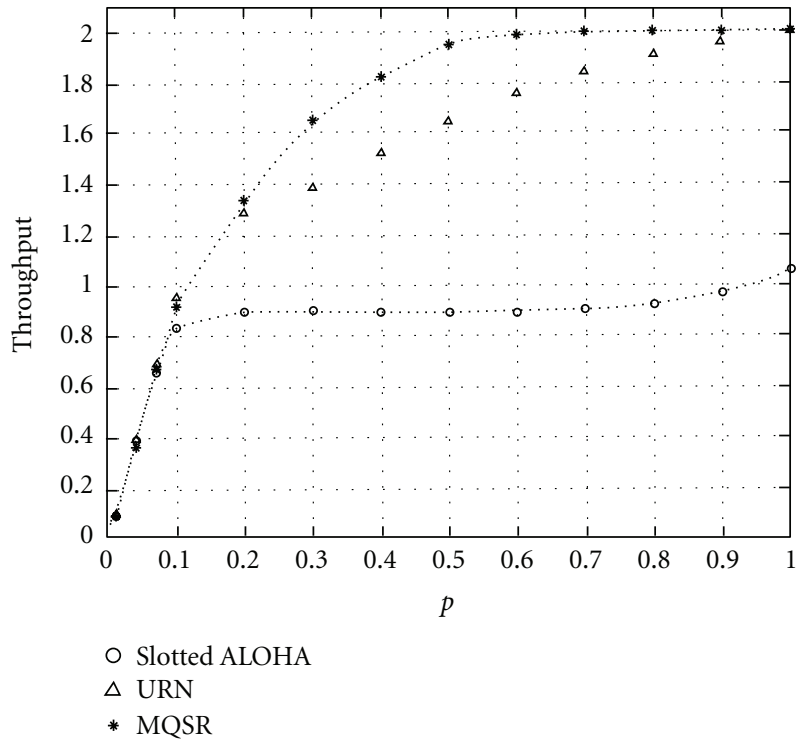

FIGURE 5: Throughput of MQSR.

MPR capability. To overcome this problem, they added an alternative model to the MAC protocol in which a node decreases its transmission probability following success and increases it following failure. This approach makes no sense in the conventional collision model, but was proved to outperform the standard model in terms of both throughput and fairness in a network with MPR capability. They also present a modified contention window technique as the easier implementation of GDP protocol. The most important contribution of $[17,46]$ is that a MAC protocol designed for MPR may diverge from the traditional backoff mechanisms in random access.

Reference [18] proposed a dynamic multi-group priority queueing protocol to exploit the cooperative diversity for improving the system throughput. Cooperative diversity is a well-established notion in MIMO systems; it can be also applied on MAC protocol design for MPR. The proposed cooperative multi-group priority queueing MAC protocol has two independent parts, one in the base station and the other in the wireless nodes, so it is based on a server-client model, which is not scalable.

4.2. Network-Layer Adaptation. Wang and Garcia-LunaAceves analyzed the performance upper bound of joint routing and scheduling for wireless ad hoc networks that embrace interference by using MPR [2]. They propose a polynomial-time heuristic algorithm to approximate the optimal solution which consists of route selection followed by link scheduling. By maximizing the number of nodedisjoint multipaths, the throughput can better approximate the performance upper bound (Table 3). A simple example to illustrate is given in Figure 6 .

In case Figure 6(b), two links can be scheduled to transmit in each time slot and fully exploit the MPR capability.

The routing and link scheduling in MPR is still an open topic. To fully exploit MPR, appropriate routing algorithm and scheduling schemes should be designed. A set of parameters could be optimized, such as schedule length or number of simultaneous paths in the network.

In [19], Crichigno et al. address the minimum length scheduling problem in one-hop multiaccess networks. They define the capacity region as the closure of the convex hull of a set of rate vectors. Figure 7 gives an example of the capacity region extension of 2-MPR over a single user channel. The function $\varphi i(P / \eta)$ denotes the channel capacity for a given reception power $P$ and a given channel noise $\eta$. The upper bound capacity is achieved at sum rate, and the length of a schedule is minimized when concurrent transmitters operate at these sum points. They propose a linear program for the Minimum Length Scheduling Problem (MLSP) which their representation defines.

Reference [20] studies the neighbor discovery algorithm by incorporate multi-packet reception capability. Jeon and Ephremides consider neighbor discovery by incorporating MPR and Physical-Layer signal processing. They adopt the viewpoint of random set theory (RST) to propose a method for estimating the set of transmitting neighbors.

Almost simultaneously, Zeng et al. studied the time for achieving neighbor discovery on an arbitrary node with MPR [21]. For an idealized MPR network, the time for all the nodes to discover their respective neighbors is $\Theta(\ln n)$ when a simple Aloha-like algorithm is used. With a more realistic $k$-MPR model, they show that the time to discover all neighbors is $\Theta(n \ln n / k)$. Table 4 lists the discussed works related to networking. A trend of a cross-layer approach to better exploit the MPR advantages is introduced by these works. In the next part, we detail an example realized on a network with scheduling.

4.3. Scheduling for Multihop Routing with MPR. Reference [2] proposed to maximize the number of node-disjoint 


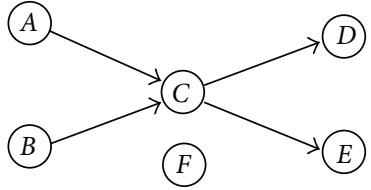

(a) Nondisjoint multipath

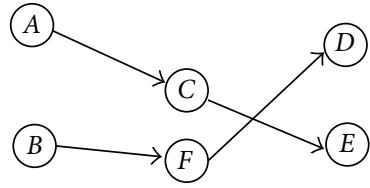

(b) Node-disjoint multipath
FIGURE 6: Influence of node-disjoint routing on link scheduling in [2].

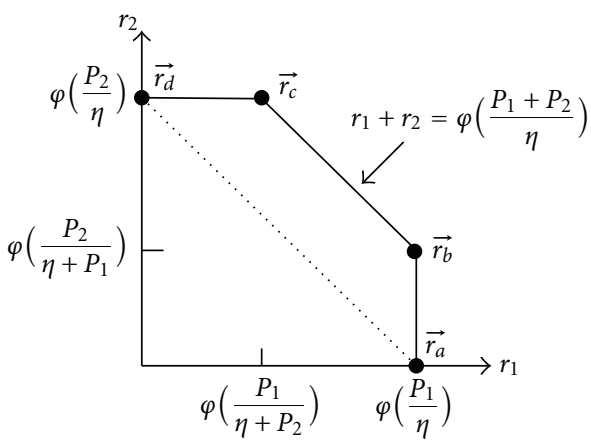

Figure 7: Capacity region of a multiaccess channel for a receiver node receiving data from two simultaneous transmitters.

TABLE 4: Networking with MPR.

\begin{tabular}{llc}
\hline Protocol & Problem & Works \\
\hline Wang's & Joint routing and scheduling & {$[2]$} \\
HS for LP-MLSP & Maximum length of scheduling & {$[19]$} \\
RST-based estimation & Neighbor discover & {$[20]$} \\
Zeng's & Neighbor discover & {$[21]$} \\
\hline
\end{tabular}

multi-paths with joint routing and scheduling, but by using node-disjoint paths, at any time slot, each receiver only receives one packet for relaying. The intermediate nodes in routing paths cannot be effective MPR receivers. Figure 8 gives an example with two data flows ( $A$ to $D$ via $C$, and $B$ to $E$ via $C$ ). Ideally, $C$ could benefit from its $2-\mathrm{MPR}$ to receive simultaneously from $A$ and $B$ at slot 1 and use the next two slots to transmit the received packets to $D$ and $E$. But with node-disjoint paths, it requires four slots to transport two flows. This example also shows that the intermediate nodes in a wireless network might become bottlenecks for throughput.

Adopting the notation of the one-hop scheduling problem as defined in [19], the channel capacity for a K-MPR receiver is

$$
\varphi_{v}=\varphi\left(\frac{\sum_{i \in S_{v}} P_{i v}}{\eta}\right)
$$

where $\varphi_{v}$ is the channel capacity of the receiver $v$ for a given reception power $P_{v}$ on it. For a general number of transceivers, the sum of transmission rates is within the

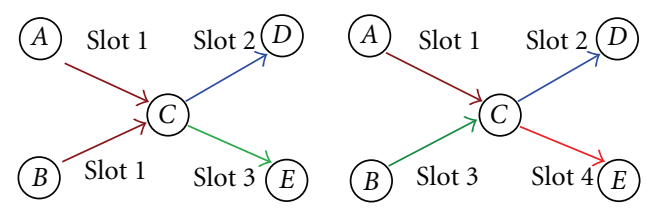

FIgURE 8: node-disjoint multipath is not optimal for MPR.

channel capacity given in (11). Therefore, we have the following inequality:

$$
\sum_{i \in S_{v}} r_{i v} \leq \varphi_{v}\left(\frac{\sum_{i \in S_{v}} P_{i v}}{\eta}\right),
$$

where $r_{i v}$ denotes the transmission rate on $i$ to $v$.

For $M$ data flows, we denote the source and the destination of $m$ th flow $s_{m}$ and $d_{m}$. The flow rate on a directed link $(u, v)$ is denoted by $f_{u v}^{m}$. It is worth noting that this flow rate is an average rate and the transmission rate $r_{u v}^{m}$ could be much higher for an intermediate receiver. For instance, if node $v$ decodes $k$ packets from different transmitters including $u$ and takes $t_{r}$ slots to relay the received packets, then the relation between flow rate $f_{u v}^{m}$ and transmission rate $r_{u v}^{m}$ can be expressed as

$$
\frac{1}{f_{u v}^{m}}=\frac{t_{r}+1}{r_{u v}^{m}}
$$

4.3.1. Problem Formulation of TMP. We formulate the Throughput Maximizing Problem (TMP) for multi-flow multi-hop communications in [47] as follows.

Definition 1. Maximize the sum of flow rate reaching all destinations:

$$
\operatorname{Maximize} \sum_{m=1}^{M} \sum_{i} r_{i d_{m}}^{m}
$$

subject to the following three constraints:

(1) flow conservation constraint:

$$
\sum_{i} r_{i j}^{m}=\sum_{j} r_{j i}^{m} ; \quad \sum_{i} r_{s_{m}}^{m}=\sum_{i} r_{i d_{m}}^{m} ;
$$

(2) receiver constraint:

$$
\forall v \in \rho, \quad\left|S_{v}\right| \leq K
$$

(3) transmitter constraint:

$$
\sum_{i \in S_{v}} r_{i v}=\varphi\left(\frac{\sum_{i \in S_{v}} P_{i v}}{\eta}\right) .
$$

We have adopted the flow conservation constraint (15) from [2]. However, the other two constraints are different. 
Receiver Constraint. A receiver $v$ cannot decode more than $K$ packets at the same time, and hence the number of transmitters in $S_{v}$ should be limited to $K$ for any slot. This constraint covers the receiver's pair-wise interference.

Transmitter Constraint. Each transmitter should operate at sumrate to fully explore the bandwidth of the receiver's multiuser channel, as given in (11).

By resolving TMP as an optimization problem, we can obtain a performance upper bound. Similar problems have been shown to be NP-hard $[2,48]$. The size of our optimization problem increases exponentially with the number of routing paths. Let us focus more on the computation of its upper bound with ideal time-space scheduling.

4.3.2. Heuristic Scheme with Distributed Scheduling on $k$-CDS. A heuristic approach with distributed scheduling based on a $k$-CDS backbone can approximate the upper bound. The $k$ CDS ( $k$-connected dominating set) [49] in a network is a set of nodes which is $k$-dominating and $k$-connected, meaning that every node in the network is either in the $k$-CDS or has $k$ neighbors in it. The subgraph of this node set is $k$ vertex connected. The properties of $k$-dominating and $k$ connected are a perfect match for intermediate relay nodes to exploit MPR capability, because each of them is required to collaborate with at least $K+1$ neighbors for both receptions and transmissions.

(i) If a receiver is a $k$-dominated node, then the set consisting of all its $k$-dominating nodes is the schedulable set $S$.

(ii) If a receiver is a dominating node in $k$-CDS, then the $k$-connected property guarantees that it is connected to at least $k$ dominating nodes. These nodes can be selected to form schedulable set $S$ for each reception slot.

(iii) If a transmitter is a $k$-dominated node, then it could schedule with $k$ dominating nodes to transmit.

(iv) If a transmitter is a dominating node, then it could schedule with $k$ dominating neighbors to transmit.

Based on the $k$-CDS backbone, only dominating nodes are selected as intermediate relay nodes for multi-hop routing and a dominated node does not participate in the routing unless it is the source or the destination of a flow. This simple rule reduces the complexity of design time-space scheduling in the network.

Many algorithms tend to generate a minimal $k$-CDS, but the transmission will be too concentrated to this set of nodes. On the other hand, a high cardinality means little reduction from the original network topology, which is not efficient to reduce the complexity of the scheduling based on the $(K+1)$-CDS. This tradeoff on the cardinality of a $(K+1)$ CDS can be calculated as follows. Let us assume that the average routing path length is $p l$. The dominated nodes only participate in the first-hop communications as source nodes or in the last-hop communications as destination nodes, while the dominating nodes can take part in each hop in a routing path. By assuming the scheduling has a good fairness for all nodes, the amount of flows that the dominated nodes take is approximately

$$
T(k-\mathrm{CDS})=\frac{K+2}{(K+1) *(p l-1)} .
$$

To meet the above constraints, we develop a construction algorithm based on a coverage rule [50]. Each node verifies if any pair of its neighbors are $k$-connected via nodedisjoint paths and a higher ID's rule is added to avoid mutual decision blocking. This verification is known as the $k$ Coverage condition. To realize this algorithm in a distributed and localized manner, nodes exchange their routing tables with their neighbors. The $k$-Coverage condition is checked via the routing table to count the number of node-disjoint paths from any pair of neighbors.

The $(K+1)$-CDS construction algorithm results in a backbone for multi-path routing. We present here a transmitter-receiver scheduling to fully exploit $K$-MPR capability on dominating and dominated nodes, which allows the use of multiple paths for each flow to eliminate bottlenecks on the intermediate nodes.

A potential transmitter $i$ constructs a receiver set $\xi_{i}$. The receivers are ordered in each set along with their distances to the final destination $d_{m}$ in number of hops. If $d_{m}$ belongs to $N(i)$, the neighbor set of $i$, then $\left\{\xi_{i}\right\}$ contains only $d_{m}$.

With the link scheduling algorithm, a receiver aims to let transmitters operate at a rate based on $(K+1)$-CDS. It schedules transmitter nodes with the priority $\mathrm{pr}_{i}$ in an arbitrary order. Every node's priority is set to minimal before any transmissions. A transmitter node $i$ is chosen, and checks its possible receivers set $\xi_{i}$. If the transmitter finds a receiver $v$ who can receive more flows, then it will be added in the receiver's schedulable set $S_{v}$. If the transmitter node cannot find any available receiver, then its priority $\mathrm{pr}_{i}$ will be increased. Hence, during the next time slot, the transmitter $i$ has a higher priority than other transmitters and will be added to the schedulable set sooner.

For each transmitter allowed to transmit, the algorithm selects the corresponding temporary datarate, according to the sum-rate constraint. For a schedulable set $S_{v}=$ $\left\{u_{1}, u_{2}, \ldots, u_{K}\right\}$, the corresponding data-rates are

$$
r_{1}^{\prime}=\varphi_{v}\left(\frac{P_{1}}{\eta}\right) ; \quad r_{K}^{\prime}=\varphi_{v}\left(\frac{P_{K}}{\eta+\sum_{j=0}^{K-1} P_{j}}\right)
$$

The sum of all the data rates is equal to $\varphi_{v}\left(\left(\sum_{i \in S_{v}} P_{i}\right) / \eta\right)$. Those data rates obey the sum-rate constraint, whatever the number of transmitters in schedulable set $S_{v}$. The link scheduling algorithm allows the transmitter $i$ an amount of time $t_{i}=T / K$, where $T$ is the time slot duration. This ensures that all transmitters will have the same time slot fraction to send their data. Since the first temporary data rate is much higher than the others, the channel utilization needs to be respread to the selected transmitters in order to achieve fairness and avoid generating bottlenecks on the lowrate transmitters. As a result, the overall throughput can be improved. 
The final data rates also obey the sum-rate constraint. Let $b_{i}^{m}(t)$ be the transmitter $i$ 's initial amount of data to send during time slot $t$ for the flow $m$. The amount of effectively transmitted data is $t_{i} \times r_{i}$ and hence the remaining amount of data to transmit for the flow $m$ can be represented as $b_{i}^{m}(t+1)=b_{i}^{m}(t)-t_{i} \times r_{i}$. The transmitter $i$ 's priority $\mathrm{pr}_{i}$ will be increased, if $b_{i}^{m}(t+1)$ is not equal to 0 .

4.3.3. Performance Evaluation. We set the channel bandwidth $W=1 \mathrm{MHz}$, transmission power $P_{0}=1 \mathrm{~W}$, and path loss exponent $\gamma=3$. In a square of $300 * 300,50$ transmitters are randomly generated. According to low-noise SNR condition $\left(\mathrm{SNR}_{\text {ref }}=10 \mathrm{~dB}\right), \mathrm{SNR}_{\text {ref }}=P_{0} d_{\text {ref }}^{-\gamma} / \eta$, and the maximal distance between two nodes $d_{\text {ref }}=44 \mathrm{~m}$, we can obtain that $\eta$ is equal to $1,16.10^{-6} \mathrm{~W}$.

The numerical results on upper bound of TMP are obtained through lpslove [51], a mixed linear programming solver. We simulate our heuristic-based $(K+1)$-CDS and heuristic based on node-disjoint path [2] in NetLogo4.1 simulator [52]. We performed 100 simulations with a duration of 2000 time slots. For each flow injected in the network, it has fixed source and destination. And it generates one packet per time slot in a saturation condition.

The metrics used are as follows: the throughput represents the number of flows which arrive at destinations during a predefined number of time slots; the average delay represents the difference between the moment the flow was sent and the moment it is received; the average acceptance ratio is the ratio of traffic accepted to the total traffic demand.

The overall throughput results obtained are shown in Figure 9 with a 3D representation. The throughput upper bound describes the maximal amount of occupied reception time slots at all destinations, which is independent of the number of flows. However, it is shown that it increases with MPR capability. Our heuristic based on $(K+1)$ CDS outperforms the heuristic with node-disjoint paths on almost all simulation settings. The node-disjoint heuristic reaches the limit very quickly with the increase of the number of flows, because node-disjoint paths are fewer than the routing paths on $k$-CDS. Our heuristic has a higher throughput limit, although it decreases when the number of flows is large ( 15 flows).

We also note that there is a local highest throughput for $K$-MPR. The throughput of 3 -MPR is highest. It is also confirmed in Figure 10. This is a very interesting observation that the throughput decreases when $K$ becomes bigger with both heuristics. One possible explanation is that the 4-MPR capability requires a much higher density to be fully exploited. The increase of node degree results in more links interfering with each other, which could decrease the network throughput. For our heuristic, the decrease of throughput with 4-MPR is also related to our link scheduling algorithm, particularly the way we spread the receiver's channel capacity between its transmitters. Indeed, the increase of channel capacity is not very large with MPR capability, while the amount of data to send is much higher (Figure 11).

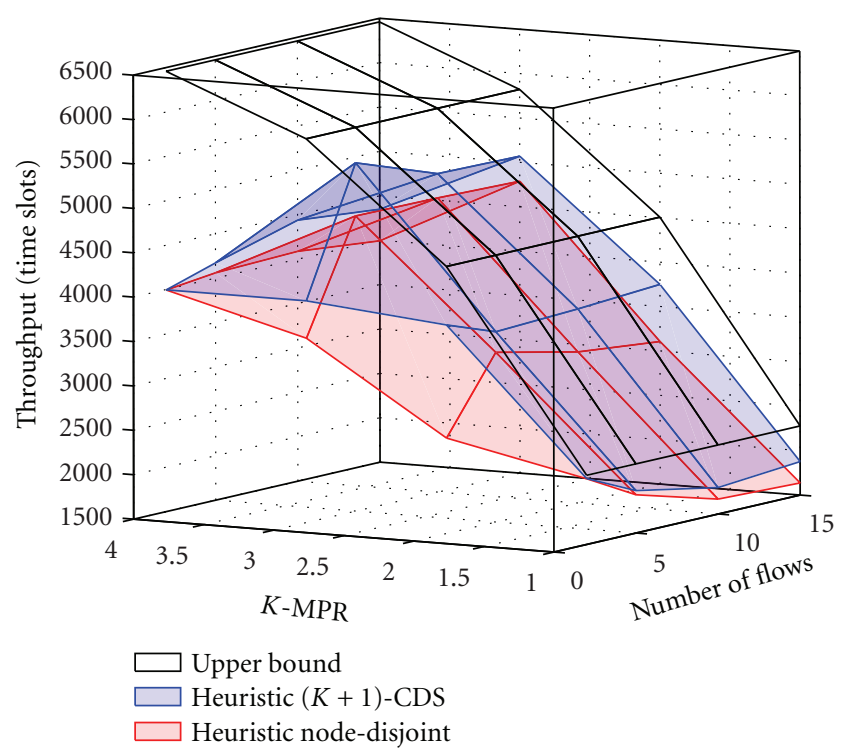

FIgURE 9: The throughput of upper bound and heuristics.

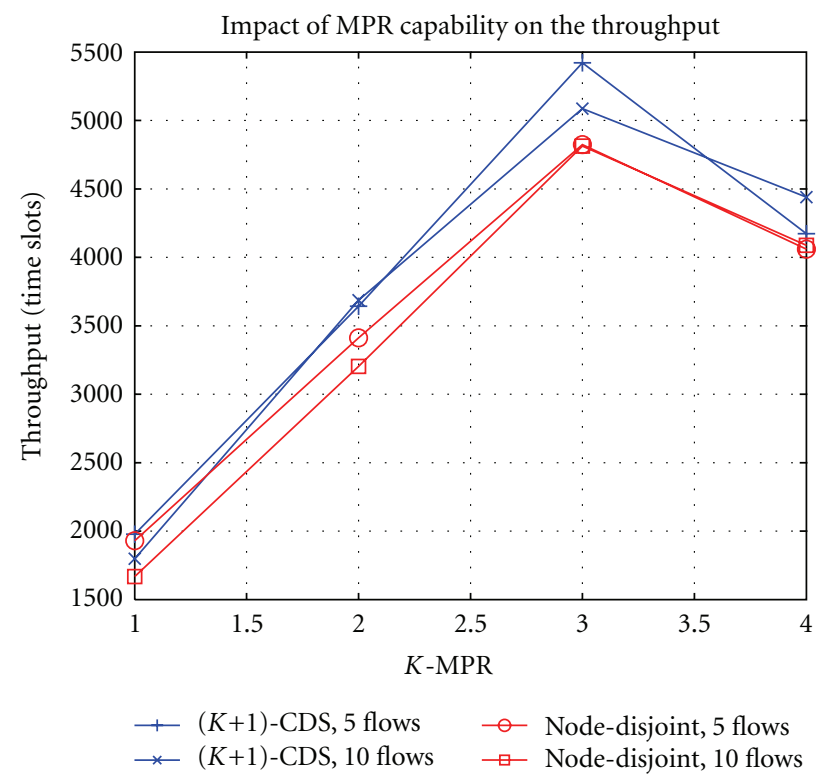

FIGURE 10: The 3-MPR can achieve the highest throughput.

Figure 12 shows that the average delay of a flow increases with the number of flows. Despite that, using MPR can reduce flow delay by around $20 \%$ compared to the single reception model. The increase of delay also confirms the presence of bottlenecks, which also cause a degradation of the flow acceptance ratio as indicated in Figure 13. Again, MPR could improve the acceptance ratio by using time-space scheduling to avoid bottleneck generation.

\section{Conclusion}

The capacity of current wireless random access networks is constrained mainly by concurrent packet transmissions. 


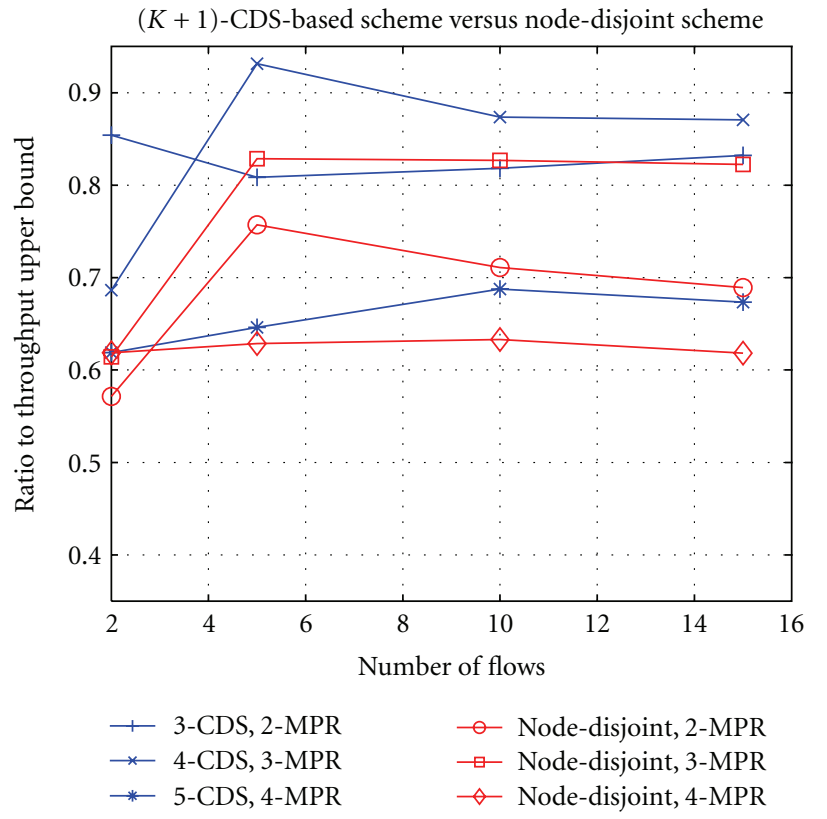

Figure 11: The heuristics' efficiency, subject to upper bound.

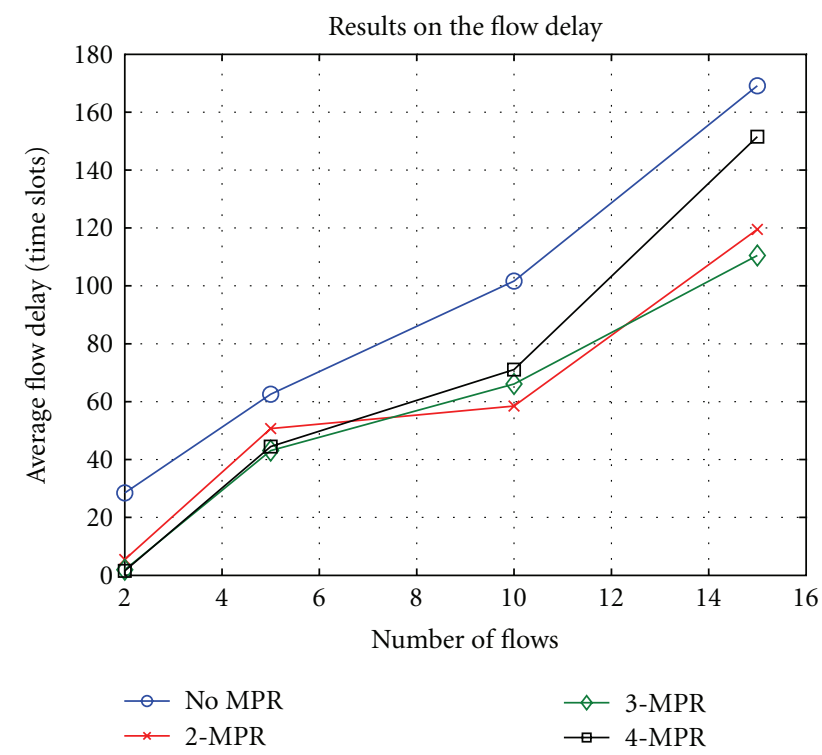

FIgURE 12: The delay increases with the number of flows.

Multi-access techniques are investigated to avoid concurrent access to the medium. MPR provides another way to shift the responsibility from the transmitters to the receivers. Originally, MPR techniques were proposed for mobile communication systems and then later introduced to wireless random access networks. Therefore, it is important to survey the techniques that enable MPR and in particular those that are applicable for wireless random access networks. This paper provides such a survey.

We also list and compare all the results on the network capacity, throughput, and stability and classify the research efforts on protocol design. Basic MPR techniques have

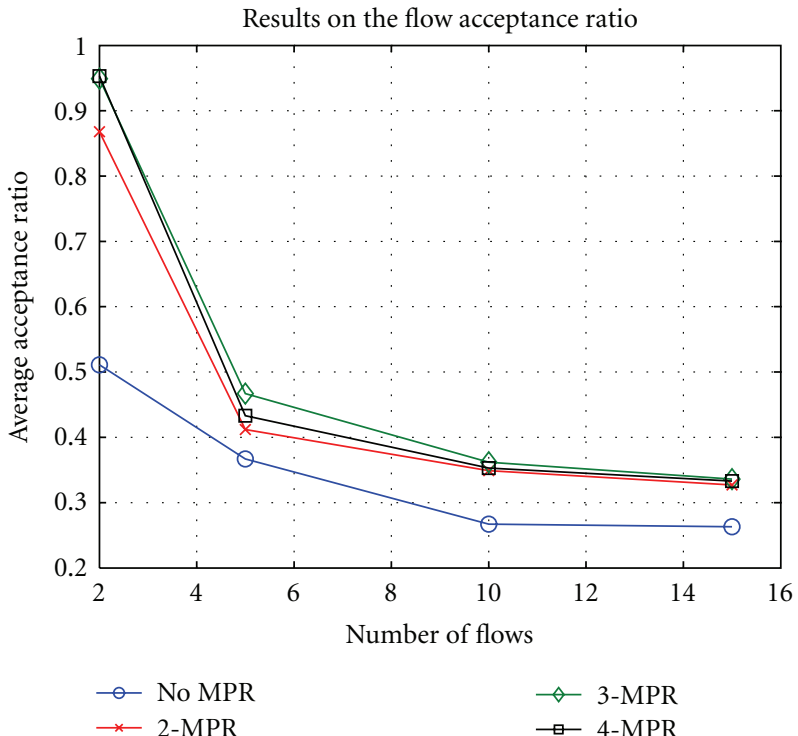

Figure 13: The flow acceptance ratio of $K$-MPR chutes.

already been applied to telecommunication services, but for wireless networks with typical random access protocols (such as MAC and Network); the protocols should be revised to fully exploit the advantage of MPR. One direction is to jointly consider routing and scheduling. We detailed such work in this paper with extensive performance comparisons. The intent of this paper is to provide a good starting point for future exploration in the area.

\section{Acknowledgments}

This research was supported by NSF of China under grant no. 60773091, no. 61100210 and Doctoral Program foundation of Institutions of Higher Education of China under Grant no. 20110073120021.

\section{References}

[1] P. X. Zheng, Y. J. Zhang, and S. C. Liew, "Multipacket reception in wireless local area networks," in Proceedings of the IEEE International Conference on Communications, vol. 8, pp. 36703675, Istanbul, Turkey, June 2006.

[2] X. Wang and J. J. Garcia-Luna-Aceves, "Embracing interference in ad hoc networks using joint routing and scheduling with multiple packet reception," in Proceedings of the 27th Int'l Conference on Computer Communications (INFOCOM '08), pp. 843-851, Phoenix, Ariz, USA, April 2008.

[3] L. Kleinrock and Y. Yemini, "An optimal adaptive scheme for multiple access broadcast communication," in Proceedings of the Int'l Conference on Communications, pp. 721-725, Torento, Canada, 1978.

[4] R. Nelson and L. Kleinrock, "The spatial capacity of a Slotted ALOHA multihop packet radio network with capture," IEEE Transactions on Communications, vol. 32, no. 6, pp. 684-694, 1984.

[5] S. Ghez, S. Verdu, and S. C. Schwartz, "Stability properties of slotted Aloha with multipacket receptionca-pability," IEEE 
Transactions on Automatic Control, vol. 33, no. 7, pp. 640-649, 1988.

[6] S. Ghez, S. Verdu, and S. C. Schwartz, "Optimal decentralized control in the random access multipacket channel," IEEE Transactions on Automatic Control, vol. 34, no. 11, pp. 1153$1163,1989$.

[7] I. Chlamtac and A. Farago, "An optimal channel access protocol with multiple reception capacity," IEEE Transactions on Computers, vol. 43, no. 4, pp. 480-484, 1994.

[8] G. Mergen and L. Tong, "Receiver controlled medium access in multihop ad hoc networks with multipacket reception," in Proceedings of the Military Communication Conference (MILCOM '01), vol. 2, pp. 1014-1018, Vienna, Austria, October 2001.

[9] M. Coupechoux, T. Lestable, C. Bonnet, and V. Kumar, "Throughput of the multi-hop slotted aloha with multi-packet reception," Wireless On-Demand Network Systems, vol. 2928, pp. 239-243, 2004.

[10] J. J. Garcia-Luna-Aceves, H. R. Sadjadpour, and Z. Wang, "Challenges: towards truly scalable ad hoc networks," in Proceedings of the 13th Annual ACM Int'l Conference on Mobile Computing and Networking (MobiCom), pp. 207-214, Montreal, Canada, September 2007.

[11] J. J. Garcia-Luna-Aceves, H. R. Sadjadpour, and Z. Wang, "Extending the capacity of ad hoc networks beyond network coding," in Proceedings of the Int'l Conference on Wireless Communications and Mobile Computing (IWCMC '07), pp. 91-96, Honolulu, Hawaii, USA, August 2007.

[12] Z. Wang, H. R. Sadjadpou, and J. J. Garcia-Luna-Aceves, "The capacity and energy efficiency of wireless Ad Hoc networks with multi-packet reception," in Proceedings of the 9th ACM International Symposium on Mobile Ad Hoc Networking and Computing, pp. 179-188, Hong Kong, China, May 2008.

[13] M. F. Guo, X. Wang, and M. Y. Wu, "On the capacity of k-MPR wireless networks," IEEE Transactions on Wireless Communications, vol. 8, no. 7, pp. 3878-3886, 2009.

[14] W. Huang, K. Letaief, and Y. Zhang, "Cross-layer multi-packet reception based medium access control and resource allocation for space-time coded MIMO/OFDM," IEEE Transaction on Wireless Communications, vol. 7, pp. 3372-3384, 2008.

[15] J. B. Seo and V. C. M. Leung, "Design and analysis of cross-layer contention resolution algorithms for multi-packet reception slotted ALOHA systems," in Proceedings of the 18th International Conference on Computer Communications and Networks (ICCCN '09), pp. 1-6, August 2009.

[16] Q. Zhao and L. Tong, "A multiqueue service room MAC protocol for wireless networks with multipacket reception," IEEE/ACM Transactions on Networking, vol. 11, no. 1, pp. 125$137,2003$.

[17] G. D. Celik, G. Zussman, W. F. Khan, and E. Modiano, "MAC for networks with multipacket reception capability and spatially distributed nodes," in Proceedings of the 27th IEEE Communications Society Conference on Computer Communications, pp. 1436-1444, Phoenix, Ariz, USA, April 2008.

[18] W. F. Yang, J. Y. Wu, and T. S. Lee, "An enhanced multipacket reception MAC protocol: cooperative approach," in Proceedings of the 3rd International Conference on Communications and Networking in China (ChinaCom '08), pp. 516-520, August 2008.

[19] J. Crichigno, M. Y. Wu, and W. Shu, "Minimum length scheduling in single-hop multiple access wireless networks," in Proceedings of the IEEE International Conference on Communications, Captown, South Africa, May 2010.
[20] J. Jeon and A. Ephremides, "Neighbor discovery in a wireless sensor network: multipacket reception capability and physical-layer signal processing," in Proceedings of the 48th Annual Allerton Conference on Communication, Control, and Computing, pp. 310-317, October 2010.

[21] W. Zeng, X. Chen, A. Russell, S. Vasudevan, B. Wang, and W. Wei, "Neighbor discovery in wireless netzorks with multipacket reception," in Proceedings of the ACM Int'l Symposium on Mobile Ad Hoc Networking and Computing (MobiHoc '11), Paris, France, May 2011.

[22] K. Sakakibara, M. Hanaoka, and Y. Yuba, "On the cusp catastrophe of slotted ALOHA systems with capture and multi-packet reception," in Proceedings of the IEEE Global Telecommunications Conference, vol. 5, pp. 3093-3098, Sydney, Australia, November 1998.

[23] L. Tong, Q. Zhao, and G. Mergen, "Multipacket reception in random access wireless networks: from signal processing to optimal medium access control," IEEE Communications Magazine, vol. 39, no. 11, pp. 108-112, 2001.

[24] M. F. Guo, X. Wang, and M. Y. Wu, "On the capacity of k-MPR wireless networks using multi-channel multi-interface," in Proceedings of the Int'l Conference on Wireless Communications and Mobile Computing (IWCMC '09), pp. 665-669, Leipzig, Germany, June 2009.

[25] Z. Wang, H. R. Sadjadpour, and J. J. Garcia-Luna-Aceves, "Fundamental limits of information dissemination in wireless ad hoc networks-part II: multi-packet reception," IEEE Transactions on Wireless Communications, vol. 10, no. 3, pp. 803813, 2011.

[26] V. Naware, G. Mergen, and L. Tong, "Stability and delay of finite-user slotted ALOHA with multipacket reception," IEEE Transactions on Information Theory, vol. 51, no. 7, pp. 26362656, 2005.

[27] A. Dua, "Random access with multi-packet reception," IEEE Transactions on Wireless Communications, vol. 7, no. 6, pp. 2280-2288, 2008.

[28] N. Pappas, A. Ephremides, and A. Traganitis, "Stability and performance issues of a relay assisted multiple access scheme with MPR capabilities," in Proceedings of the International Symposium of on Modeling and Optimization of Mobile, Ad Hoc, and Wireless Networks (WiOpt '11), pp. 110-116, May 2011.

[29] N. Pappas, A. Ephremides, and A. P. Traganitis, "RelayAssisted Multiple Access with Multi-Packet Reception Capability and Simultaneous Transmission and Reception," in Information Theory Workshop (ITW), 2011 IEEE, pp. 578-582, October 2011.

[30] R. M. D. Moraes, H. R. Sadjadpour, and J. J. Garcia-LunaAceves, "Many-to-many communication: a new approach for collaboration in MANETs," in Proceedings of the 26th IEEE International Conference on Computer Communications (INFOCOM '07), pp. 1829-1837, Anchorage, Alaska, USA, May 2007.

[31] A. J. Van der Veen and L. Tong, "Packet separation in wireless ad-hoc networks by know modulus algorithms," in Proceedings of the IEEE International Conference on Acoustic, Speech, and Signal Processing (ICASSP '02), vol. 3, pp. 2149-2152, Orlando, Fla, USA, May 2002.

[32] J. Y. Wu, W. F. Yang, L. C. Wang, and T. S. Lee, "Signal modulus design for blind source separation via algebraic known modulus algorithm: a perturbation perspective," in Proceedings of the IEEE International Symposium on Circuits and Systems, pp. 3013-3016, Seattle, Wash, USA, May 2008. 
[33] R. Babu and R. Kumar, "Blind equalization using Constant Modulus algorithm and Multi-Modulus Al-gorithm in wireless communication systems," International Journal of Computer Applications, vol. 1, no. 3, pp. 40-45, 2010.

[34] A. G. Orozco-Lugo, M. M. Lara, D. C. McLernon, and H. J. Muro-Lemus, "Multiple packet reception in wireless ad hoc networks using polynomial phase-modulating sequences," IEEE Transactions on Signal Processing, vol. 51, no. 8, pp. 2093 2110, 2003.

[35] M. K. Tsatsanis, R. Zhang, and S. Banerjee, "Networkassisted diversity for random access wireless networks," IEEE Transactions on Signal Processing, vol. 48, no. 3, pp. 702-711, 2000.

[36] X. Wang and J. K. Tugnait, "A bit-map-assisted dynamic queue protocol for multiaccess wireless networks with multiple packet reception," IEEE Transactions on Signal Processing, vol. 51, no. 8, pp. 2068-2081, 2003.

[37] S. Verdu, "Minimum probability of error for asynchronous Gaussian multiple access channels," IEEE Transactions on Information Theory, vol. 32, no. 1, pp. 85-96, 1986.

[38] A. Li, M. Wang, X. Li, and H. Kayama, "A cross-layer design on the basis of multiple packet reception in asynchronous wireless network," in Proceedings of the IEEE International Conference on Communications, pp. 3477-3484, June 2007.

[39] R. Lupas and S. Verdu, "Near-far resistance of multiuser detectors in asynchronous channels," IEEE Transactions on Communications, vol. 38, no. 4, pp. 496-508, 1990.

[40] R. M. Buehrer, N. S. Correal, and B. D. Woerner, "A comparison of multiuser receivers for cellular CDMA," in Proceedings of the Global Telecommunications Conference (Globecom '96), pp. 1571-1577, London, UK, November 1996.

[41] Y. J. Zhang, S. C. Liew, and D. R. Chen, "Delay analysis for wireless local area networks with multipacket reception under finite load," in Proceedings of the IEEE Global Telecommunications Conference (GLOBECOM '08), pp. 1-6, New Orleans, La, USA, November 2008.

[42] Y. J. Zhang, P. X. Zheng, and S. C. Liew, "How does multiplepacket reception capability scale the performance of wireless local area networks?" IEEE Transactions on Mobile Computing, vol. 8, no. 7, pp. 923-935, 2009.

[43] Y. J. Zhang, S. C. Liew, and D. R. Chen, "Sustainable throughput of wireless lans with multipacket reception capability under bounded delay-moment requirements," IEEE Transactions on Mobile Computing, vol. 9, no. 9, pp. 12261241, 2010.

[44] Y. J. Zhang, "Multi-round contention in wireless LANs with multipacket reception," IEEE Transactions on Wireless Communications, vol. 9, no. 4, pp. 1503-1513, 2010.

[45] Y. Yu and G. B. Giannakis, "SICTA: a 0.693 contention tree algorithm using successive interference cancellation," in Proceedings of the IEEE International Conference on Computer Communications (INFOCOM '05), pp. 1908-1916, March 2005.

[46] G. D. Celik, G. Zussman, W. F. Khan, and E. Modiano, "MAC for networks with multipacket reception capability and spatially distributed nodes," IEEE Transactions on Mobile Computing, vol. 9, no. 2, pp. 226-240, 2010.

[47] J. Lu, P. Vandenhove, W. Shu, and M. Wu, "Enhancing throughput in wireless multi-hop network with multiple packet reception," in Proceedings of the IEEE Int'l Conference on Communications, pp. 1-5, Kyoto, Japan, 2011.

[48] M. Kodialam and T. Nandagopal, "Characterizing achievable rates in multi-hop wireless networks: the joint routing and scheduling problem," in Proceedings of the 9th Annual
International Conference on Mobile Computing and Networking (MobiCom '03), pp. 42-54, San Diego, CA, USA, September 2003.

[49] F. Dai and J. Wu, "On constructing k-connected k-dominating set in wireless ad hoc and sensor networks," Journal of Parallel and Distributed Computing, vol. 66, no. 7, pp. 947-958, 2006.

[50] J. Wu and F. Dai, "A generic distributed broadcast scheme in ad hoc wireless networks," IEEE Transactions on Computers, vol. 53, no. 10, pp. 1343-1354, 2004.

[51] lp slove 5.5., http://lpsolve.sourceforge.net.

[52] NetLogo, http://ccl.northwestern.edu/netlogo/. 

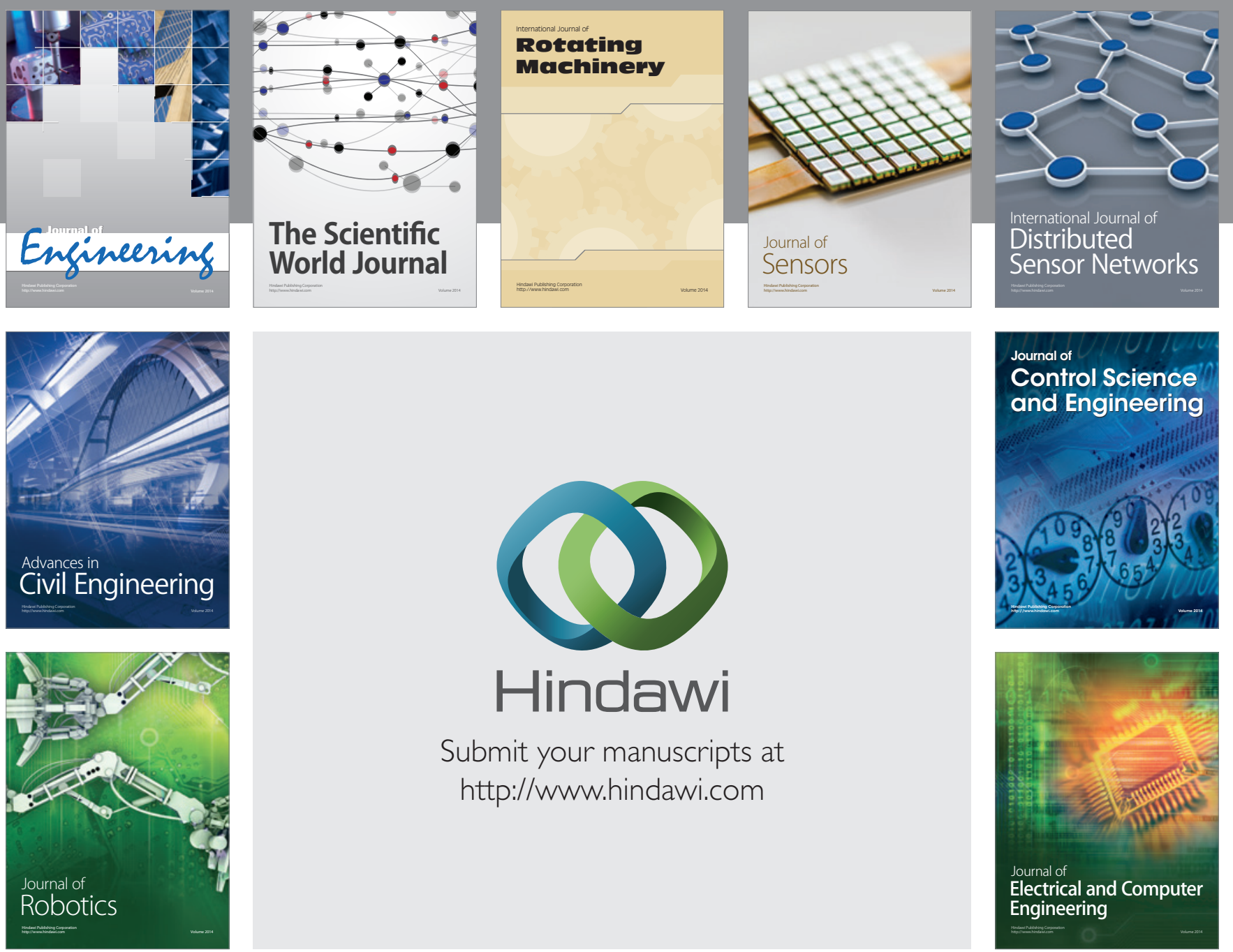

Submit your manuscripts at

http://www.hindawi.com
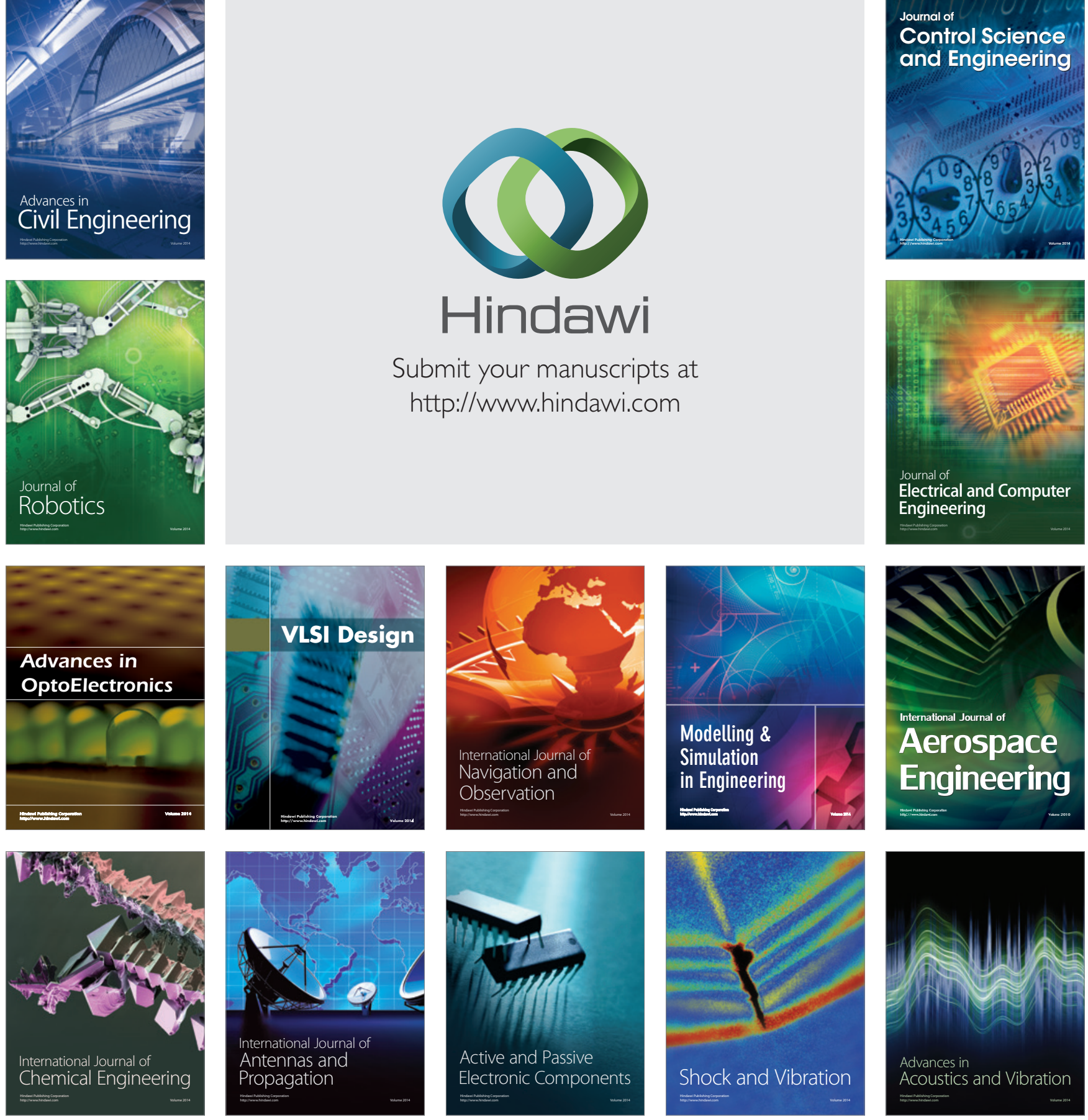